\title{
The Roles of 5G Mobile Broadband in the Development of IoT, Big Data, Cloud and SDN
}

\author{
Bao-Shuh Paul Lin1,2, Fuchun Joseph Lin',2, Li-Ping Tung1 \\ ${ }^{1}$ Microelectronics \& Information Research Center, National Chiao Tung University, Taiwan \\ ${ }^{2}$ College of Computer Science, National Chiao Tung University, Taiwan \\ Email: bplin@mail.nctu.edu.tw, fjlin@nctu.edu.tw, Iptung@nctu.edu.tw
}

Received 3 November 2015; accepted 22 February 2016; published 25 February 2016

Copyright ( 2016 by authors and Scientific Research Publishing Inc.

This work is licensed under the Creative Commons Attribution International License (CC BY). http://creativecommons.org/licenses/by/4.0/

(c) () Open Access

\begin{abstract}
The fast technology development of 5G mobile broadband (5G), Internet of Things (IoT), Big Data Analytics (Big Data), Cloud Computing (Cloud) and Software Defined Networks (SDN) has made those technologies one after another and created strong interdependence among one another. For example, IoT applications that generate small data with large volume and fast velocity will need 5G with characteristics of high data rate and low latency to transmit such data faster and cheaper. On the other hand, those data also need Cloud to process and to store and furthermore, SDN to provide scalable network infrastructure to transport this large volume of data in an optimal way. This article explores the technical relationships among the development of IoT, Big Data, Cloud, and SDN in the coming 5G era and illustrates several ongoing programs and applications at National Chiao Tung University that are based on the converging of those technologies.
\end{abstract}

\section{Keywords}

5G, Internet of Things (IoT), Software Defined Networks (SDN), Big Data Analytics, Cloud Computing

\section{Introduction}

The roles of SDN, Cloud, IoT, and Big Data in 5G Networks have raised great interest recently [1]. According to the IEEE Computer Society (IEEE CS 2022 Report [2]), Cloud Computing (Cloud), Big Data Analytics (Big Data), Internet of Things (IoT), and Software Defined Networks (SDN) are among 4 of 20+ emerging technologies as illustrated in Table 1. In the meantime, based on the report in IEEE Communications Society (IEEE ComSoc Technology News [3]), among top 10 trends in 2015 as listed in Table 2, 5G, Virtualization (SDN \& NFV), everywhere connectivity for IoT \& IoE, and Big Data are also included. Combining both reports, we can identify 5G, Cloud, IoT/IoE, Big Data, and SDN as the five most worthwhile ICTs (information \& communica- 
Table 1. IEEE computer society 2022 report.

\begin{tabular}{|c|c|c|}
\hline \multicolumn{3}{|c|}{ Technology \& Indexing Terms } \\
\hline 1. Security Cross-Cutting Issues & 9. Multicore & 17. 3D Printing \\
\hline 2. Open Intellectual Property Movement & 10. Photonics & 18. Big Data and Analytics \\
\hline 3. Sustainability & 11. Networking and Interconnectivity & $\begin{array}{l}\text { 19. Machine Learning and } \\
\text { Intelligent Systems }\end{array}$ \\
\hline 4. Massively Online Open Courses & 12. Software Defined Networks & 20. Life Sciences \\
\hline 5. Quantum Computing & 13. High Performance Computing & $\begin{array}{l}\text { 21. Computational Biology and } \\
\text { Bioinformatics }\end{array}$ \\
\hline 6. Device and Nanotechnology & 14. Cloud Computing & 22. Robotics \\
\hline 7. 3D Integrated Circuits & 15. Internet of Things & \\
\hline 8. Universal Memory & 16. Natural User Interfaces & \\
\hline
\end{tabular}

Table 2. IEEE communications society top 10 trends 2015.

\begin{tabular}{ll}
\hline 1) & 5G \\
2) & FIBER EVERYWHERE \\
3) & VIRTUALIZATION, SDN \& NFV \\
4) & EVERYWHERE CONNECTIVITY FOR IoT \& IoE \\
5) & BIG DATA, COGNITIVE NETWORKS \\
6) & CYBERSECURITY \\
7) & GREEN COMMUNICATIONS \\
8) & SMARTER SMARTPHONES, CONNECTED SENSORS \\
9) & NETWORK NEUTRALITY, INTERNET GOVERNANCE \\
10) & MOLECULAR COMMUNICATIONS \\
\hline
\end{tabular}

tions technologies) to watch out up to 2020 in term of their potential, convergence and applications. Figure 1 depicts the overlapping of these five major ICT trends. It also illustrates how the infrastructure deployment of 5G mobile broadband and the architectural integration of Cloud Computing strongly impact the development of IoT, Big Data, and SDN. This paper explores the technical relationships of these five areas and discusses several ongoing programs and applications currently under development at NCTU based on these technologies.

\section{The Roles of 5G, IoT, Big Data, Cloud, and SDN till 2020}

Although so far the 5G mobile broadband requirements and standard specifications are not ready yet, 5G technology research \& development are already started and some 5G features or subsystems are readily available. By Year 2020 the commercial 5G will be available and IoT applications will be deployed everywhere with mobile broadband technology. Moreover, the Big Data generated by IoT applications will become a norm and Cloud will be largely utilized to compute, store and virtualize network functions (NFV). Also, the underlying network infrastructure will adopt SDN to reduce both capital expense (CAPEX) and operational expense (OPEX). Figure 2 further illustrates the roles of 5G, IoT, Big Data, Cloud and SDN and their relationships. This figure is modified based on the reference [4] by inserting $5 \mathrm{G}$ mobile broadband in the center.

\section{Technical Relationships among IoT, Big Data, Cloud, \& SDN in 5G Era}

Based on Figure 2, we develop Figure 3 to better explain the technical relationships among IoT, Big Data, Cloud, and SDN in the 5G mobile broadband services (5G MBS) [5]. First, IoT is capable of generating Big Data with four Vs: volume, velocity, variety and veracity. Then, Cloud is brought in for Big Data storage and processing. Finally, SDN is employed to provide more efficient and flexible networks for inter-Cloud data transport. Out of Big Data, Cloud, and SDN, advanced technologies such as machine learning analytics, Cloud 


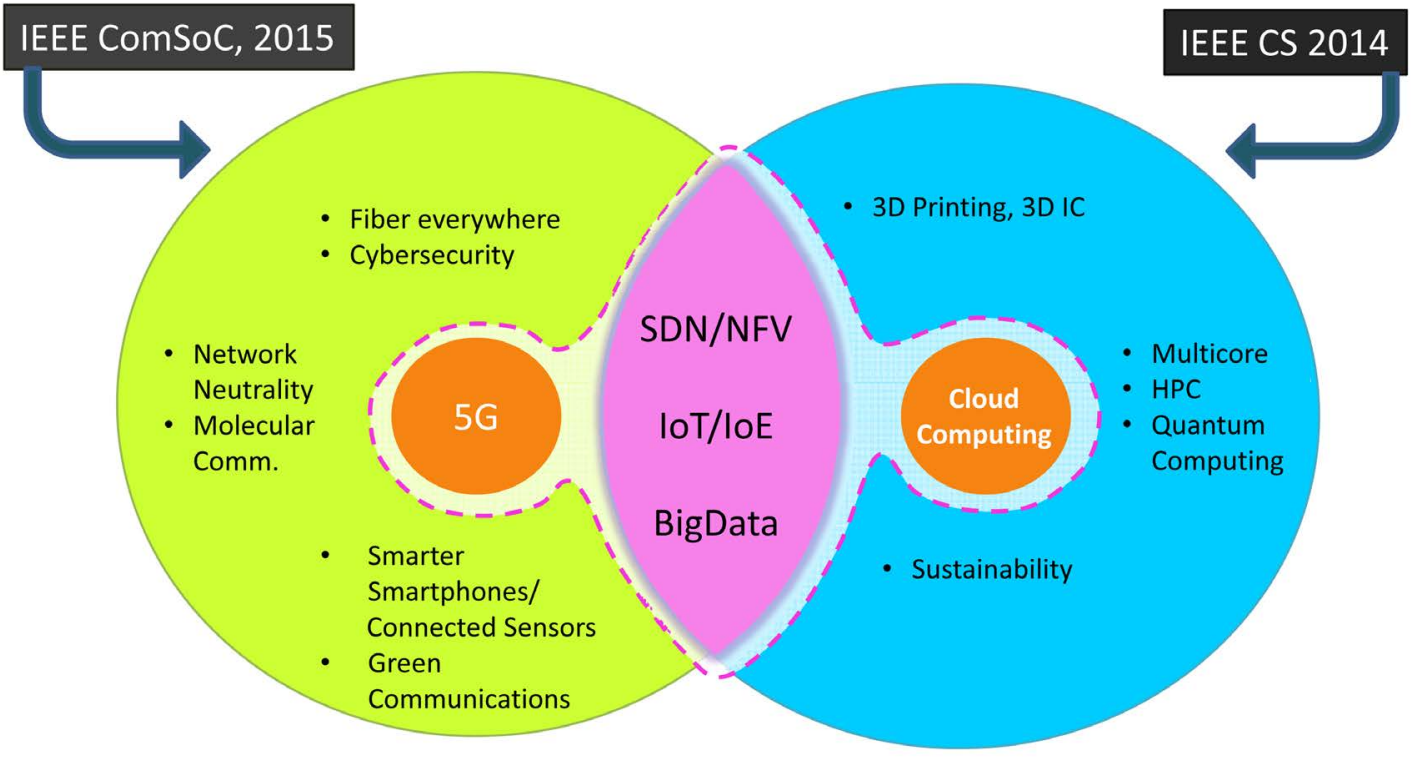

ICT : Information \& Communication Technology

Figure 1. ICT major trends for 2015-2020.

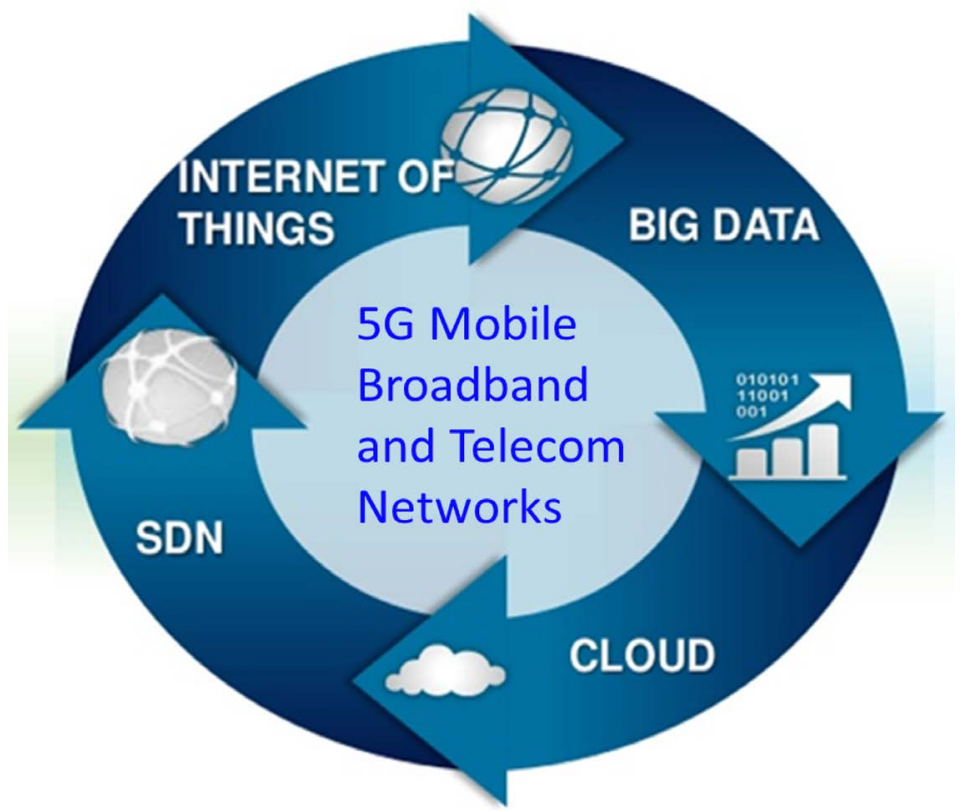

Figure 2. Relationship among 5G, IoT, Big Data, Cloud and SDN based on [4].

RAN and softwarized 5G then are developed.

As illustrated in Figure 4, 5G will serve as a better gateway and transport network for IoT applications so that IoT data can be delivered more efficiently and economically. In addition, IoT will become one of the major sources of Big Data by producing large volume, fast velocity, and many varieties of data [6] as illustrated in Figure 5.

Finally, Figure 6 shows that Cloud can be adopted in the 5G Radio Access Network (RAN) and turns it to a Cloud-based RAN (C-RAN). Both SDN and NFV have been applied to data center in the cloud to enable better load balance and resource allocation of the cloud. SDN has also been applied to 5G mobile broadband core networks to enable smart routing, better traffic management and improve network resource utilization. 


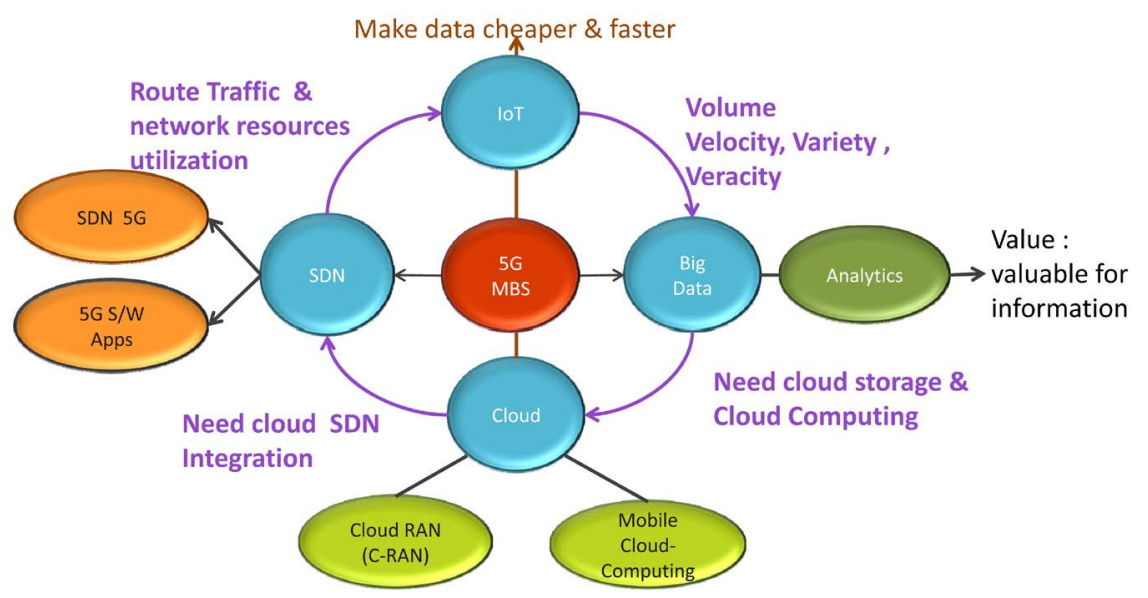

Figure 3. Technical relationships among 5G \& IoT, Big Data, Cloud, and SDN.

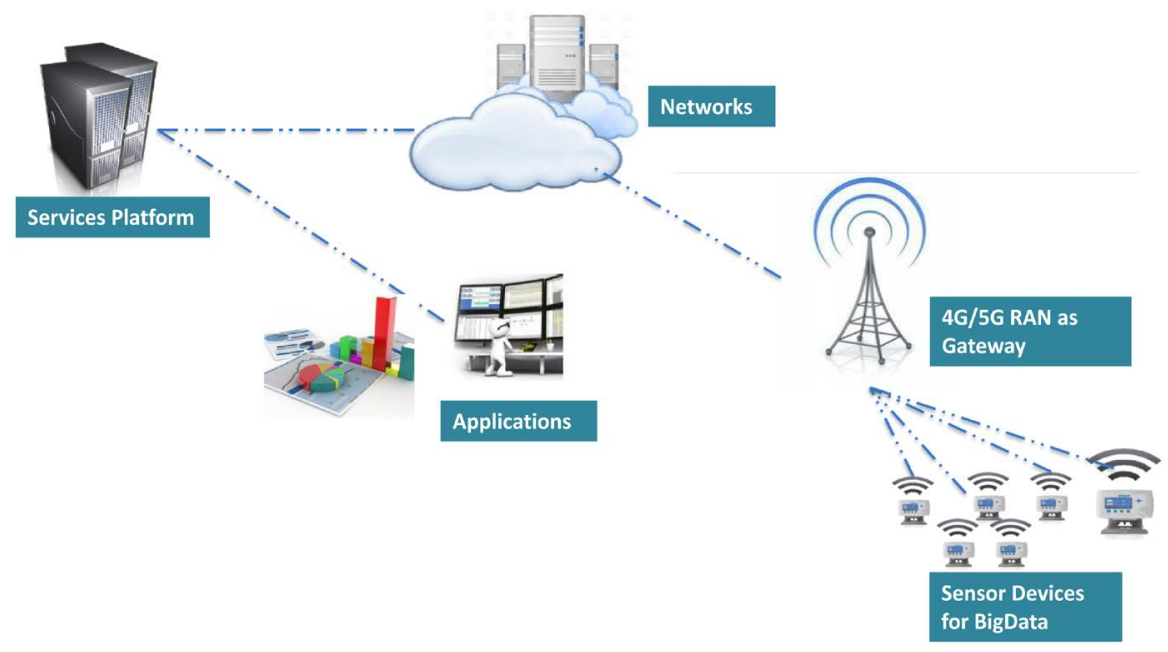

Figure 4. 4G/5G as the gateway for IoT applications.

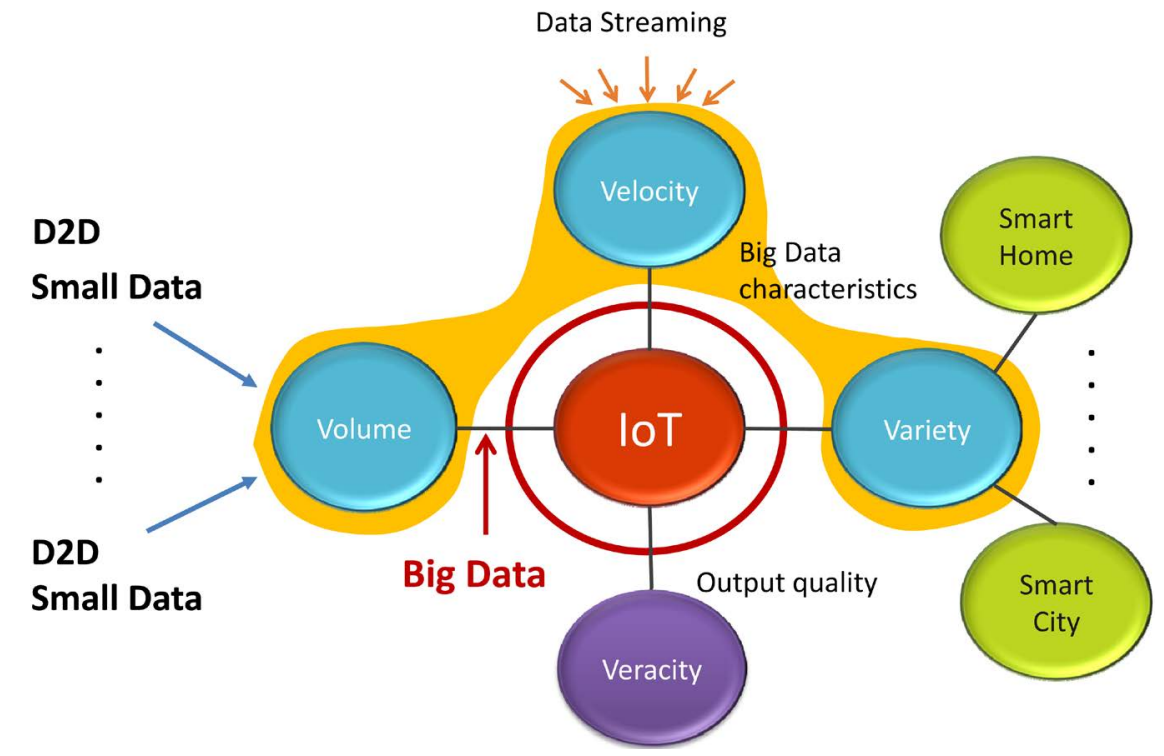

Figure 5. Relationships between IoT and Big Data. 


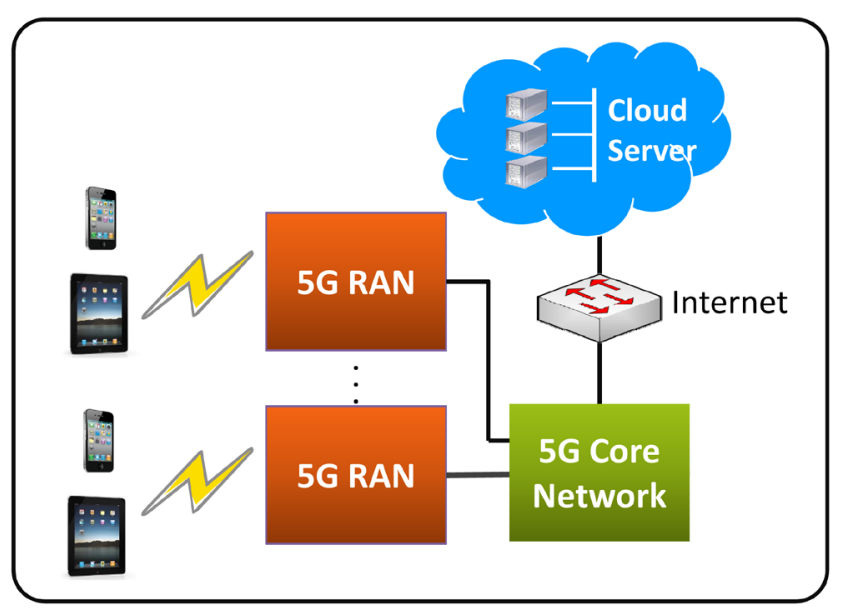

Figure 6. C-RAN architecture in 5G mobile network.

\section{Ongoing Programs and Applications at National Chiao Tung University (NCTU)}

In this section, we describe five ongoing programs and applications at NCTU that are closely related to the SDN, Cloud, Big Data, IoT, and 5G technologies [7]-[17].

\subsection{Program on SDN-Enabled Cloud-Based Broadband and Wireless Network Technologies and Services}

This program is a national program to support the SDN Industry-Academia Cooperation, led by both NCTU and CHT (Chunghwa Telecom, the largest mobile \& telecom operator in Taiwan) with 5 other networking and communications companies in Taiwan. This is a multi-year program with the target to set up a testbed for endto-end testing and to help establish an ecosystem for local SDN industry.

Figure 7 shows the scope of this program that covers mobile access, WiFi access, broadband core and data center cloud end-to-end application systems.

Figure 8 further shows both the structure of the program and its network configuration across NCTU, CHT and another university National Tsing Hwa University (NTHU).The program includes research and development involving 4G/LTE, B4G/5G, SDN, Cloud, SDN for Wi-Fi, and SDN for WAN technologies. There are five sub-programs: 1) SDN broadband network technologies and services, 2) SDN mobile/wireless network technologies and services, 3) SDN and cloud integration services and management, 4) SDN switch-related devices and systems and 5) SDN system integration and field trials.

The program is intended to construct an SDN network in NCTU, CHT and NTHU, respectively. These SDN networks then will be interconnected to form a wide area SDN network in Taiwan. Eventually, we plan to have this end-to-end system connected to the global network. The SDN switches deployed in this testbed will be mostly small or medium scale due to their experimental nature. Also included in this testbed are SDN-based Wi-Fi access points. Cloud-based data centers will be deployed in each location of NCTU, CHT and NTHU. Three types of SDN controllers including OpenDaylight, Ryu and Floodlight are currently under trial in the experimental network. Our architecture assumes the scenario of multiple SDN controllers with a hierarchical topology on which useful SDN APPs such as load balancer, network optimizer for video delivery, network coordinator (including visualization), dynamic flow configuration, end-to-end service configuration, dynamic policybased traffic engineering, and multitenant network automation can be developed and deployed.

\subsection{Program on Big Data Analytics for Network Traffic and Management Data}

In this program, we address the network performance issues with two experimental networks: BML at NCTU campus and ITRINET of ITRI.

The architecture of the BML experimental network is illustrated in the lower part of Figure 9 that consists of a 4G RAN, a 4G Core and a Cloud environment. For the 4G RAN, both indoor and outdoor environment are taken into consideration. The left upper part of Figure 9 shows the scope of the ITRINET experimental network 


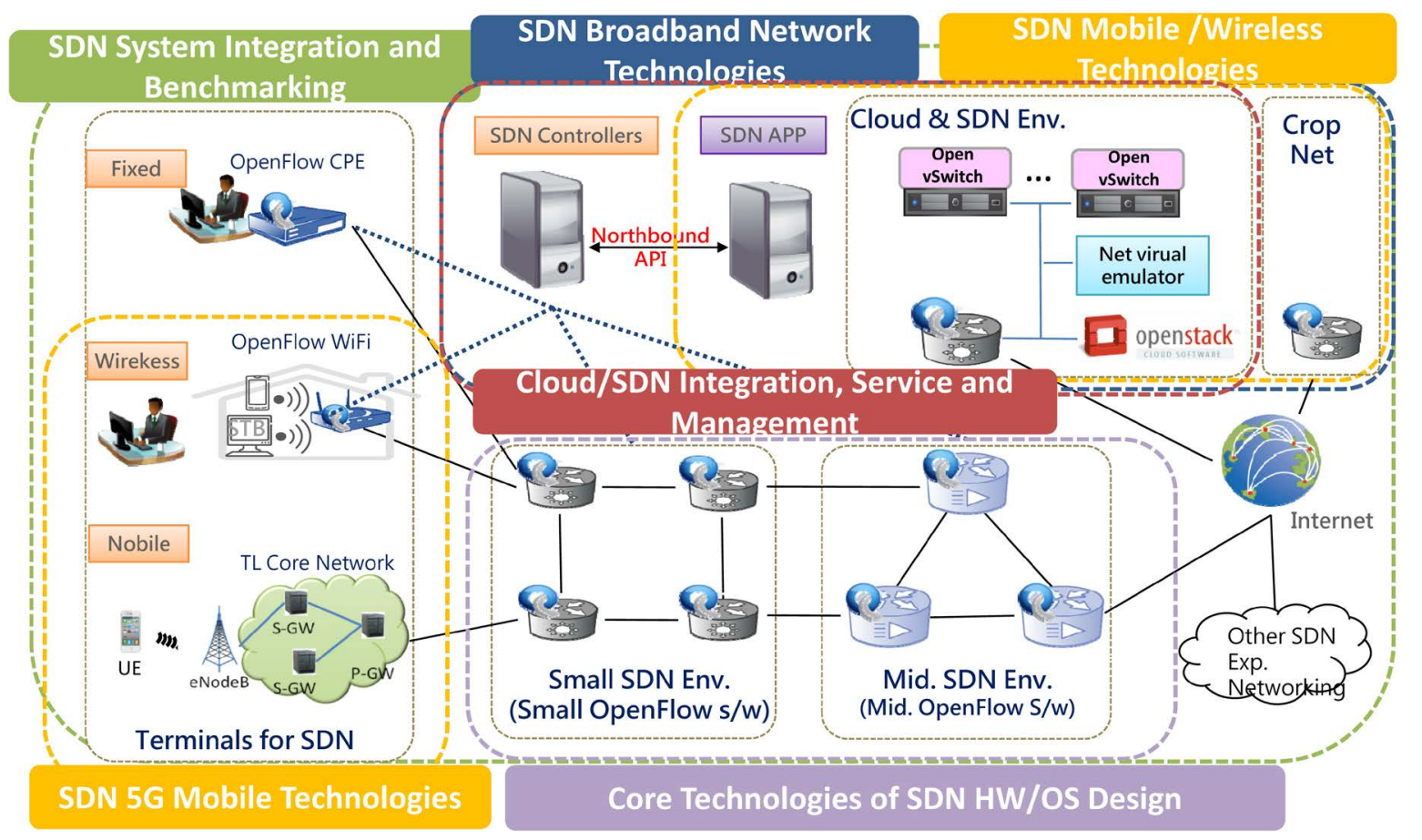

Figure 7. Applications of SDN \& cloud in 4G/LTE \& 5G wireless networks.
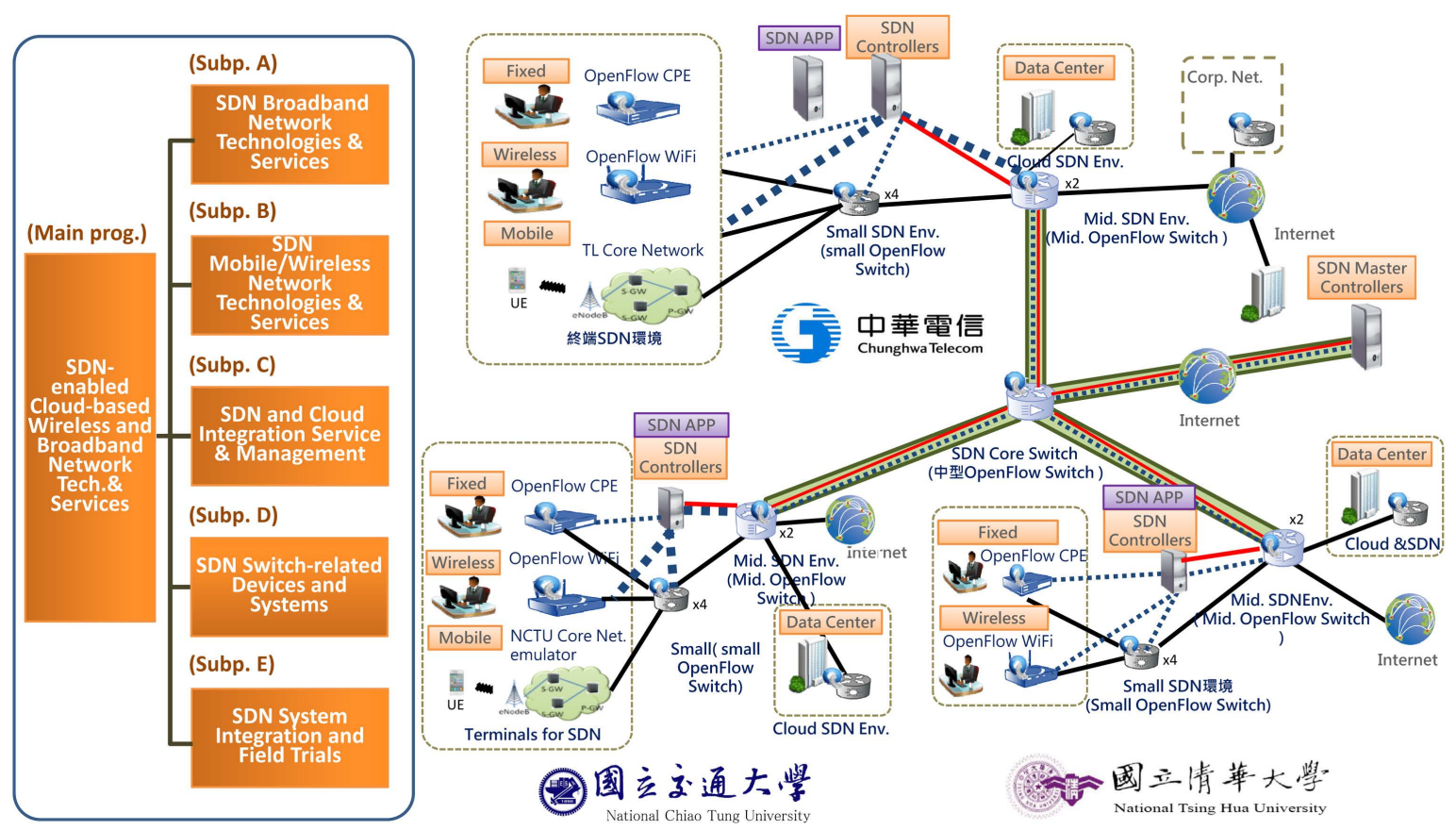

Figure 8. SDN technical R\&D center and projects at NCTU.

that covers a much greater area of Hsinchu County than NCTU and includes additionally ITRI (Industrial Technologies Research Institute), HSIP (Hsinchu Science Industrial Park) and THR (Taiwan High-speed Rail, Hsinchu station). The right upper part of Figure 9 illustrates how ITRINET covers various R\&D buildings in the corporate campus of ITRI such as B11, B12, B51, etc.

Figure 10 illustrates how Big Data analytics based on InfoSphere or Spark is performed on ITRINET for the purpose of network optimization. First, network traffic measurement and network management data are collected. 


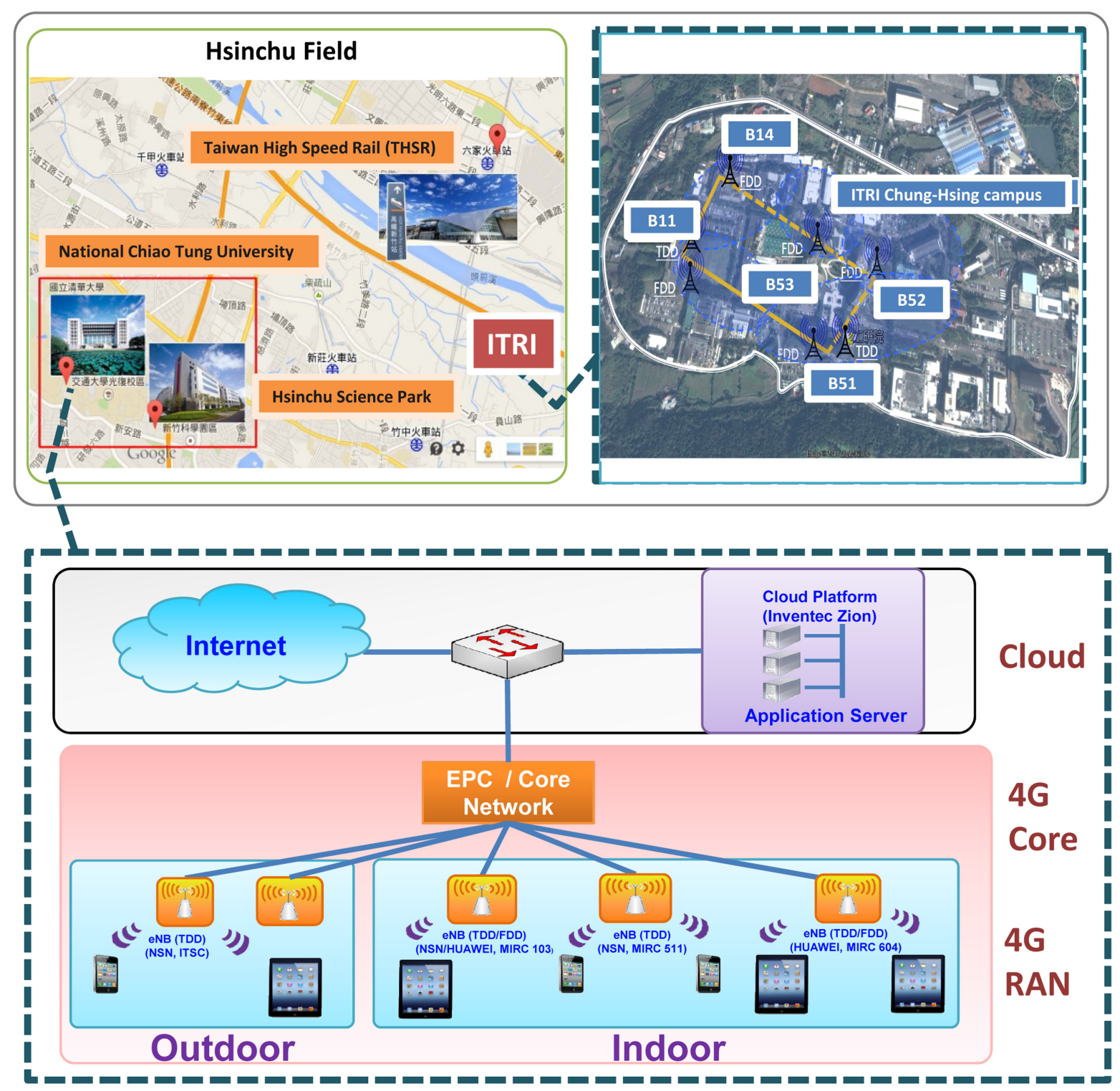

Figure 9. Greater Hsinchu 4G/LTE \& Future 5G experimental network ITRINET (include ITRI, NCTU, THR \& HSIP).

Then, data analytics methods based on machine learning, data mining and statistical modeling are applied to analyze the collected data. Finally, we apply the results thus generated to network performance evaluation and optimization by providing a feedback loop for system re-configuration. The whole operational cycle includes the technologies of 4G/LTE, B4G/5G, Big Data Analytics, Cloud, and Traffic Engineering.

\subsection{Application on IoT Platform Integrated with Data Generation and Data Analytics}

This is an application where we set up an IoT platform integrated with data generator and data analytics capabilities as illustrated in Figure 11. A common challenge for IoT/M2M service providers is how to test their large scale IoT/M2M applications with the near realistic data that the system will handle in a production environment. As such tests may involve not only a large number but also a large variety of sensors, deploying a testing environment that contains all the necessary sensors turns out to be an infeasible, if not impossible job. To tackle this problem, we develop a data generation method (illustrated in the lower part of Figure 11) based on streams generation capabilities of IBM InfoSphere and Spark to emulate data from a large number and a large variety of sensors. Such generated data will be sent into and processed by the applications residing on the IoT/M2M 


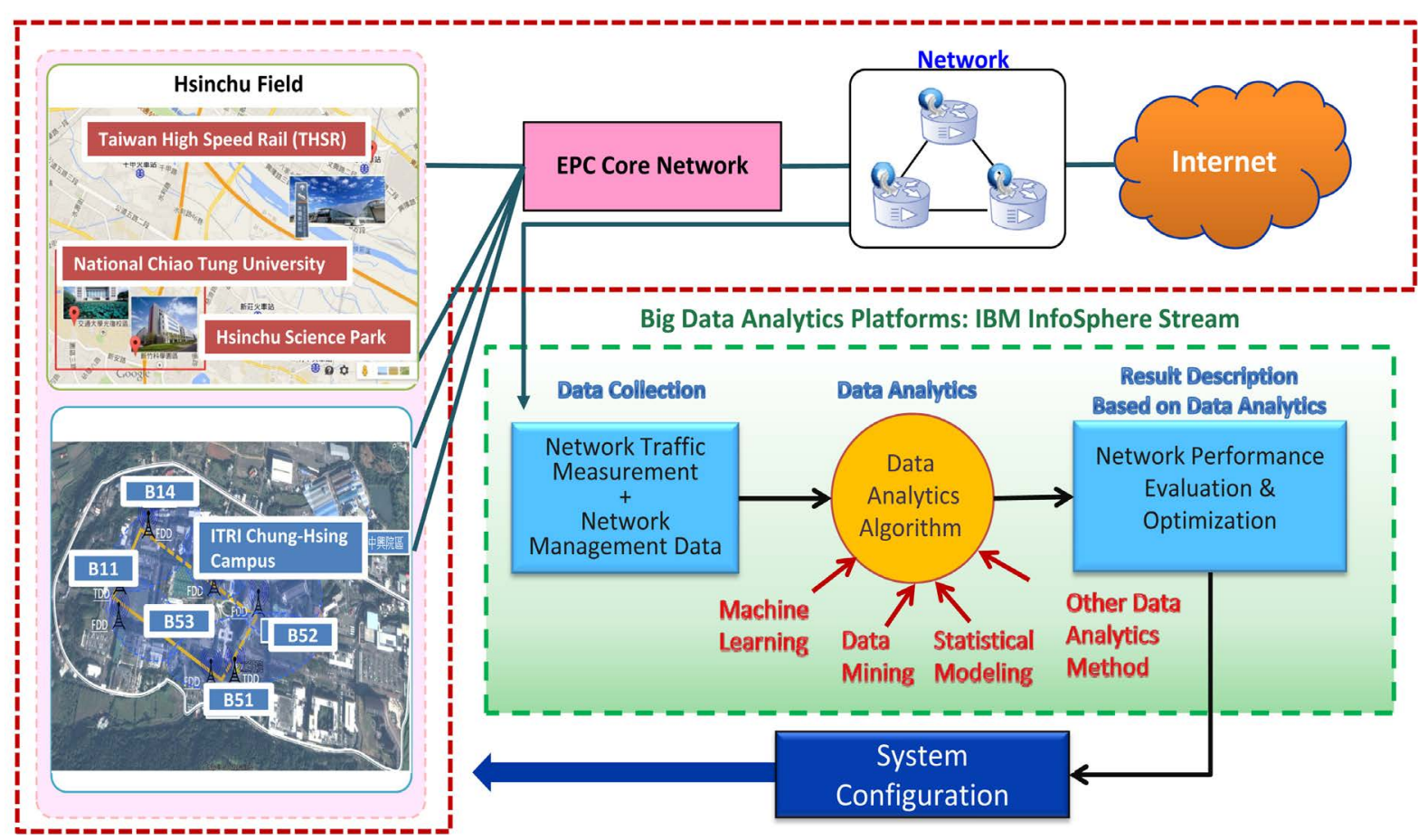

Figure 10. Big Data analytics for Traffic engineering of ITRINET.

\section{Analyzing Results from IoT/M2M Applications}

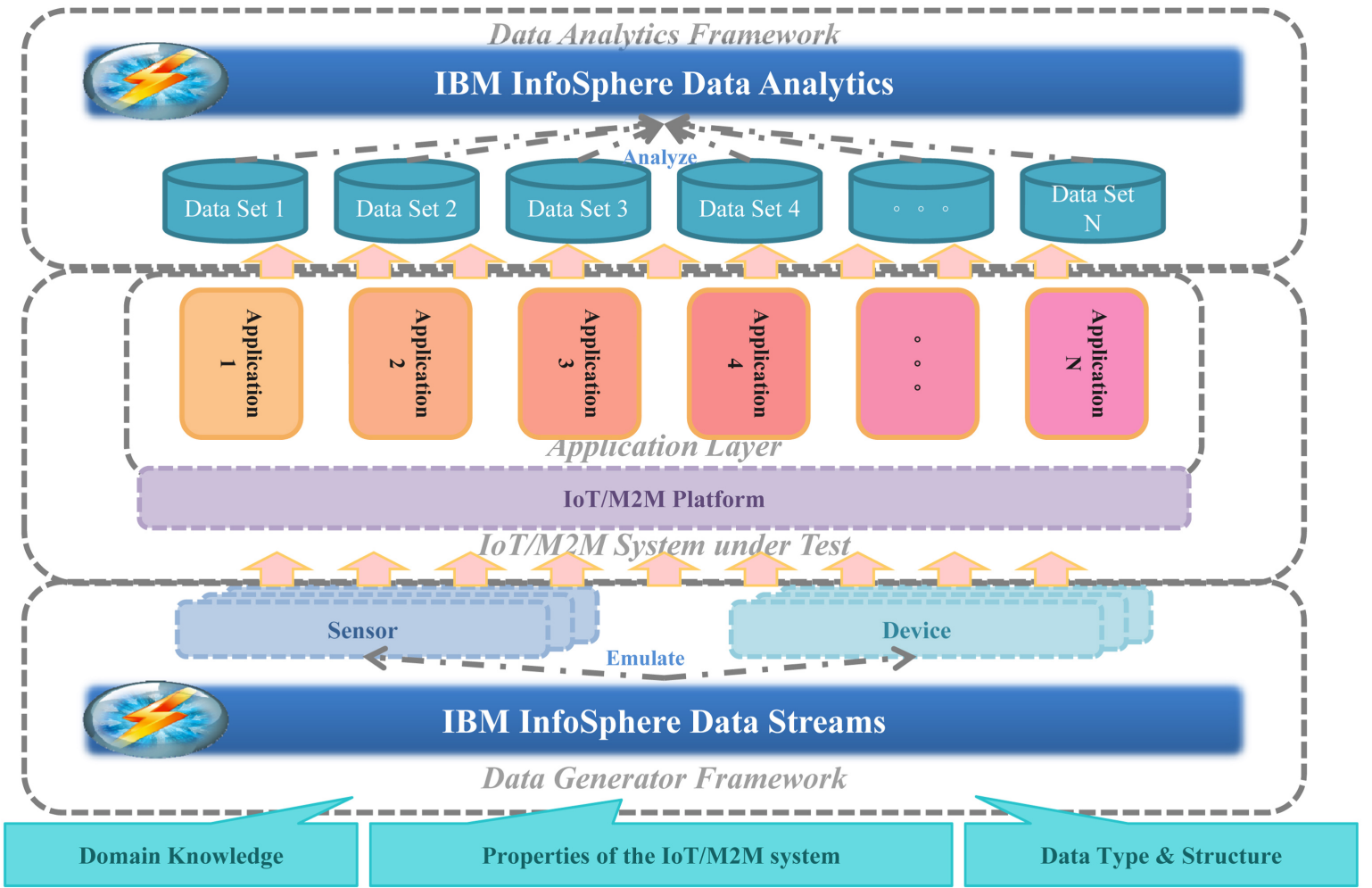

Generating Large Volume and Fast Velocity Streaming Data

Figure 11. IoT platform integrated with data generation and data analytics. 
platform (illustrated in the middle part of Figure 11). Finally, the data sets produced by the IoT/M2M applications are analyzed by data analytics engines in IBM InfoSphere and Spark (illustrated in the upper part of Figure 11).

The effectiveness of our data generation methodology is first verified in a people management system of a large factory environment. With our methodology, we are able to test our factory management IoT/M2M application more efficiently without actually deploying a large number and a large variety of sensors. This application development uses both IoT and Big Data Analytics.

\subsection{Application on Data Analytics for Traffic Generated by Testing \& Measurement}

The automatic Testing \& Measurement (T\&M) of DUT (Device under Test, including 4G/LTE SD, UE/CPE) for 4-Stage (namely, conformance, interoperability, operator-IOT and field trials) testing is shown in Figure 12.

We apply the data analytics learning techniques for the classification of the DUT types by series numbers or id. Once the type is identified, the parameters for feature extraction can be measured by the testing and measurement equipment. An example for DUT classification is shown in Table 3. In this example, the DUT type relational table is a $4 \times 4$ table with 4 fields: 1) DUT Device Type, 2) UE capability Information (FGI), 3) DNS (TTL), and 4) HTTP (User Agent). This device relational table is created by applying multivariate statistical analytics on the network traffic flow and LTE UE connection related information. Then by extracting specific features, we are able to identify the types of DUT such as Dongle, Smartphone, CPE, etc.

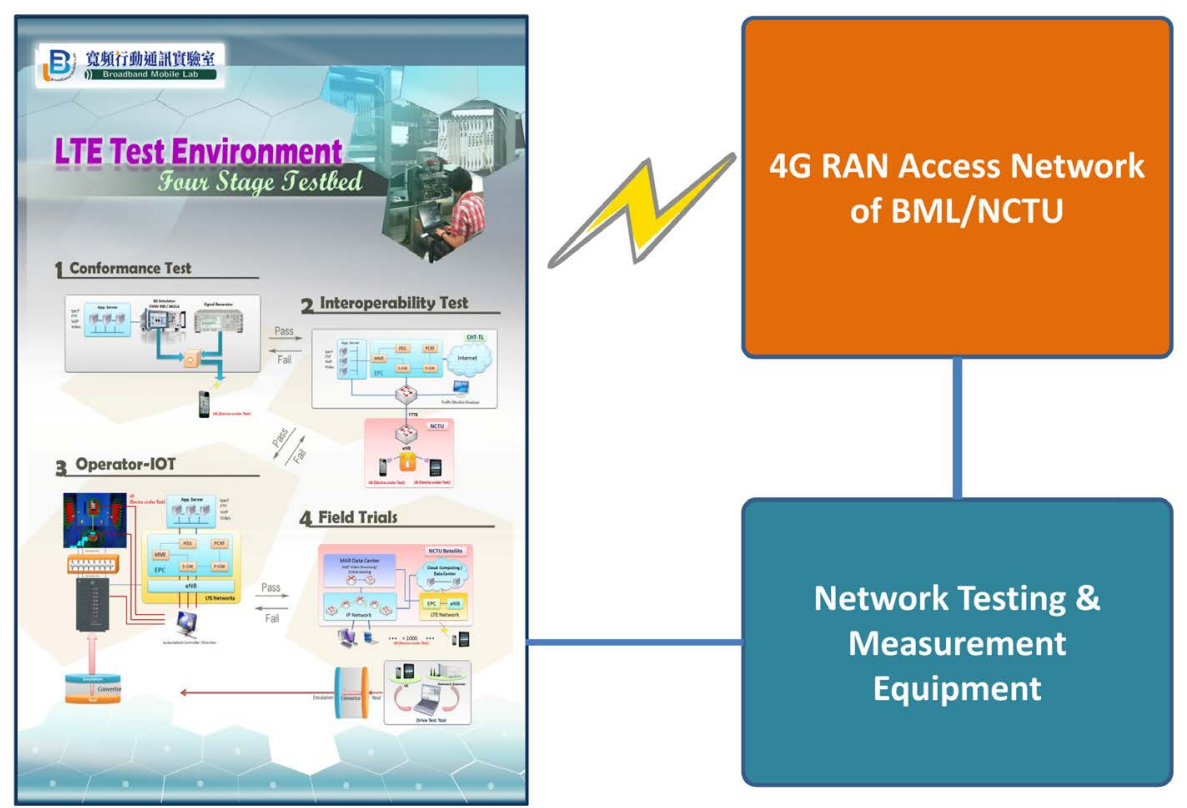

Figure 12. Data generation by endpoints from testing \& measurement.

Table 3. DUT relational table.

\begin{tabular}{|c|c|c|c|}
\hline DUT Device Type & UE FGI & DNS-TTL & HTTP-User Agent \\
\hline Dongle A & $\begin{array}{c}01000110000001010001 \\
100000000000\end{array}$ & 128 & $\begin{array}{c}\text { Mozilla/5.0 (Windows NT 6.1; WOW64) } \\
\text { AppleWebKit/537.36 (KHTML, like Gecko) } \\
\text { Chrome/43.0.2357.134 Safari/537.36\r\n }\end{array}$ \\
\hline Dongle B & $\begin{array}{c}01011111000011111111 \\
110010000000\end{array}$ & 63 & $\begin{array}{c}\text { Mozilla/5.0 (Windows NT 6.1; WOW64) } \\
\text { AppleWebKit/537.36 (KHTML, like Gecko) } \\
\text { Chrome/43.0.2357.134 Safari/537.36\r\n }\end{array}$ \\
\hline Smart Phone & $\begin{array}{c}01011110000011011101 \\
100010000000\end{array}$ & 43 & iPhone6,2/8.3 (12F70) \r\n \\
\hline CPE & $\begin{array}{c}01011111000011111111 \\
110010000000\end{array}$ & 127 & $\begin{array}{c}\text { Mozilla/5.0 (Windows NT 6.1; WOW64) } \\
\text { AppleWebKit/537.36 (KHTML, like Gecko) } \\
\text { Chrome/43.0.2357.134 Safari/537.36\r\n }\end{array}$ \\
\hline
\end{tabular}


Another example of applying data analytics in T\&M traffic is to evaluate LTE QoS. When evaluating LTE QoS, it is required to setup testing scenarios with different QoS levels. Traffic Flow Template (TFT) (as shown in Table 4) is designed to filter packets into correspondent bearers, either a default bearer or dedicated bearer, in LTE network. Each bearer has its own QoS level. We can establish a dedicated bearer of guarantee bit rate (GBR) for applications such as VoIP, or a default bearer of basic QoS level for applications like file transfer.

What TFT does is to filter packets into correspondent bearers according to packets' IP address, port number, protocol, direction. The information, however, is insufficient to differentiate among web file download, FB news browsing, and Line chat. We are unable to give different QoS levels to these applications. To tackle this problem, we propose a new architecture which integrates deep packet inspection (DPI) with TFT to provide a higher granularity of QoS levels for applications. When some traffic flow has been identified as a certain application type, TFT will be informed to update rules of packet filters. With this improved method, the traffic flow can be delivered through the bearer of suitable QoS level.

\subsection{Applications on Data Analytics for Traffic Flow Created by APP's of SD}

To fulfill the Quality of Service (QoS) requirements from users, it is important to make effective use of the network resource. We can optimize the performance of a network by applying data analytics to traffic engineering. In particular, it is important to classify mobile applications traffic intended by the user with data analytics.

We propose a HMM-based (Hidden Markov Model) model to classify the mobile applications. By surveying related work, we have realized that there are different handshake patterns of well-known application protocols. Also, according to the observation of some specific mobile applications, we discover that every mobile Internet service has its unique negotiation process at the beginning when service starts. HMM was widely used to recognize time-dependent sequences and find out unknown patterns of data, such as speech recognition, handwriting recognition.

Table 4. Traffic Flow Template (TFT): Use to specify the packet filters associated.

\begin{tabular}{|c|c|c|c|c|c|c|c|c|c|c|c|}
\hline $\begin{array}{l}\text { UL } \\
\text { Packet } \\
\text { Filter } \\
\text { ID }\end{array}$ & DRB & $\begin{array}{c}\text { Packet } \\
\text { Filter } \\
\text { Evaluation } \\
\text { Precedence }\end{array}$ & $\begin{array}{c}\text { Protocol } \\
\text { Number } \\
\text { (IPv4)/ } \\
\text { Next } \\
\text { Header } \\
\text { (IPv6) }\end{array}$ & $\begin{array}{c}\text { Remote } \\
\text { Address } \\
\text { and Subnet } \\
\text { Mask }\end{array}$ & $\begin{array}{l}\text { Single } \\
\text { Local } \\
\text { Port } \\
\text { (UE) }\end{array}$ & $\begin{array}{c}\text { Local Port } \\
\text { Range (UE) }\end{array}$ & $\begin{array}{l}\text { Single } \\
\text { Remote } \\
\text { Port } \\
\text { Range } \\
\text { (NW) }\end{array}$ & $\begin{array}{c}\text { Remote } \\
\text { Port } \\
\text { Range } \\
\text { (NW) }\end{array}$ & $\begin{array}{c}\text { IPSec } \\
\text { SPI } \\
\text { Range }\end{array}$ & $\begin{array}{c}\text { Type of } \\
\text { Service } \\
\text { (IPv4)/ } \\
\text { Traffic } \\
\text { Class } \\
\text { (IPv6) and } \\
\text { Mask }\end{array}$ & $\begin{array}{c}\text { Flow } \\
\text { Label } \\
\text { (IPv6) }\end{array}$ \\
\hline 1 & DRB2 & 6 & 6 (TCP) & $\begin{array}{c}\text { IPv4: } 172.168 .8 .0 \\
\text { [255.255.255.0] } \\
\text { IPv6: } \\
\text { 2001:0ba0::[ffff:ffff::] }\end{array}$ & 60051 & - & - & - & - & - & - \\
\hline 2 & DRB2 & 2 & 17 (UDP) & - & - & - & 60201 & - & - & - & - \\
\hline 3 & DRB3 & 7 & 6 (ТCP) & $\begin{array}{c}\text { IPv4: } 172.168 .8 .0 \\
\text { [255.255.255.0] } \\
\text { IPv6: } \\
\text { 2001:0ba0::[ffff:ffff::] }\end{array}$ & - & 60100:60200 & - & - & - & - & - \\
\hline 4 & DRB3 & 3 & $\begin{array}{c}17 \\
\text { (UDP) }\end{array}$ & - & - & - & - & $\begin{array}{l}\text { 60300: } \\
60400\end{array}$ & - & - & - \\
\hline 5 & DRB3 & 5 & $\begin{array}{l}50 \text { IPSec } \\
\text { (ESP) }\end{array}$ & - & - & - & - & - & 0x0F80F0000 & - & - \\
\hline 6 & DRB3 & 1 & - & - & - & - & - & - & - & $\begin{array}{c}00101000 \\
\text { Mask = } \\
11111100\end{array}$ & - \\
\hline 7 & DRB3 & 6 & - & - & - & - & - & - & - & - & - \\
\hline 8 & $\begin{array}{c}\text { DRB1 } \\
\text { (default } \\
\text { bearer) }\end{array}$ & 255 & $\begin{array}{c}6 \\
\text { (TCP) }\end{array}$ & - & - & - & - & - & - & - & - \\
\hline
\end{tabular}


In our method, we extract the packet size sequence and packet transmission direction sequence of the first 20 packets to train the HMM model. Figure 13 shows our designed model structure of HMM. $X_{n}$ is the hidden variable. It represents the transmission states which cannot be observed directly. Because of the unknown transmission states, we need to use observation variable $O_{n}^{1}$ and $O_{n}^{2}$ as training features to build the mobile application models. Here, " $n$ " is the sequence number of packets in a traffic flow. $O_{n}^{1}$ is the observation symbol of packets transmission direction. $O_{n}^{2}$ is the observation of packet size that quantizes to a certain range of packet size. We quantize smallest packet size to number 1 and largest packet size to number 8 . The rest of packet sizes are then divided into six groups.

Figure 14 shows the main process of our classification system. First, we process the collected packets by reading from original PCAP files, and extract the necessary field of packet header, including source IP, destination IP, source port number, destination port number, packet timestamp, and packet size. Second, we use packet

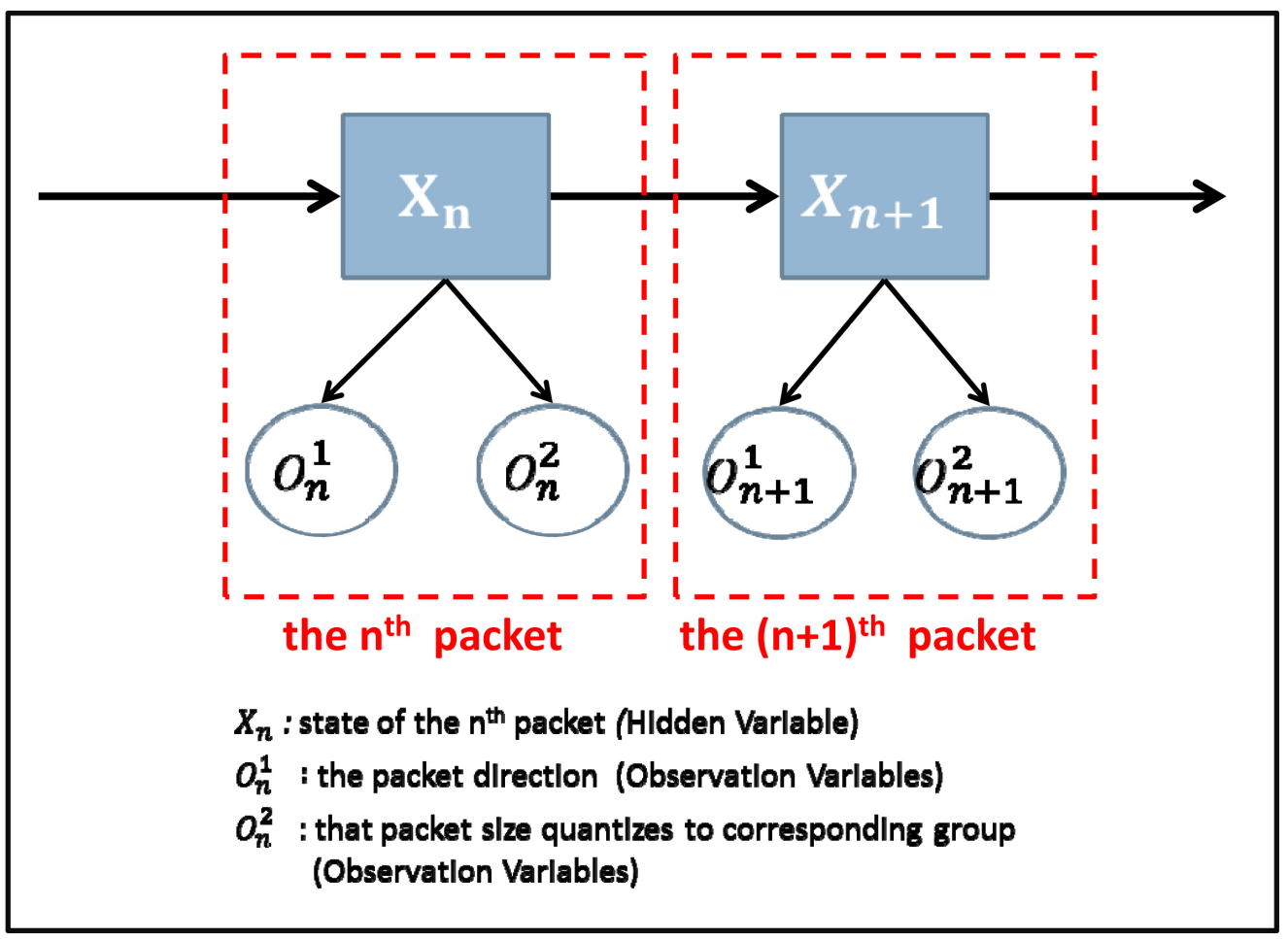

Figure 13. Classification model of Internet application traffic.

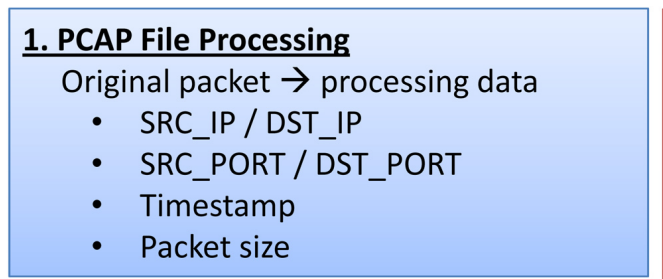

2. Feature Extraction

First 20 packets of each flow

- Packet Direction

1: Client $\rightarrow$ Server

2: Server $\rightarrow$ Client

- Packet size

Quantize to corresponding group
3. Learning Process

HMM Parameter Estimation

- Using EM algorithm

Application Training Model

- Each application has its own model

$\lambda^{1}=\left\{\pi_{1}, A_{1}, B_{1}\right\} \ldots \lambda^{N}=\left\{\pi_{N}, A_{N}, B_{N}\right\}$

\section{Recognizing Process}

Find Maximum Probability

- Observation of testing data: $O^{\text {test }}$

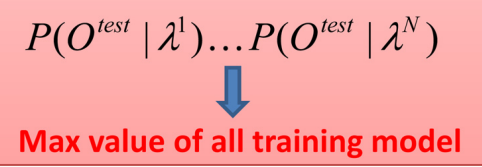

Figure 14. The process of our proposed classification system. 
header information (5-tuple: source IP, destination IP, source port number, destination port number, and protocol) to process packets with the same 5-tuple information into a unit of mobile application traffic flow, and extract the features of each application flow. Third, in learning process, we use the extracted features which are quantized into corresponding symbol sequence to training the HMM-based application models for different applications. Finally, we can identify the new traffic flow by finding out the maximum value of log likelihoods derived from different application models.

\section{Conclusion}

This paper presents a forward looking view of the convergence of IoT, big data, cloud, SDN technologies along with the arrival of 5G mobile broadband networks. We intend to demonstrate the technical relationships of those technologies and the compelling programs and applications that can be created when two, three or more of those technologies converge. Due to the nature of fast evolution of ICT and the ongoing innovation of those five technologies, this paper should be updated on annual basis to keep the related information up to day with the ICT major trends.

\section{Acknowledgements}

This work was supported by the Ministry of Science and Technology (MOST) of Taiwan and National Chiao Tung University under grants: MOST 103-2622-E-009-012-, 103-2221-E-009-138-, 103-2218-E-009-032-, 104-2221-E-009-023- and NCTU-ICTL $104 \mathrm{Q} 707$.

\section{References}

[1] Lin, B-S.P. (2015) The Roles of SDN, Cloud, IoT, \& Big Data, in 5G Networks and Their Technical Relationships \& Applications. Invited Talk, IEEE VTS APWCS 2015, Singapore.

[2] Alkhatib, H., et al. (2015) IEEE CS 2022 Report. IEEE Computer Society, IEEE.

[3] Neira, E.M. (2015) IEEE ComSoc CTN Special Issue on Ten Trends That Tell Where Communication Technologies are Headed in 2015.

[4] Tucker, L. (2013) On the Cloud of the Future. Cloud Computing Summit, San Jose.

[5] Kleiner, T. (2015) 5G Research in Horizon 2020.

[6] Thadani, A. and Andreoli, P. (2014) Realizing Business Value from Convergence of IoT + Big Data Technologies. 2014 Midwest Architecture Community Collaboration (MACC) Conference, Minneapolis, 13 November 2014.

[7] Dinh, H.T., Lee, C., Niyato, D. and Wang, P. (2013) A Survey of Mobile Cloud Computing: Architecture, Applications, and Approaches. Wireless Communications and Mobile Computing, 13, 1587-1611. http://dx.doi.org/10.1002/wcm.1203

[8] Lin, B-S.P., et al. (2013) The Design of Cloud-based 4G/LTE for Mobile Augmented Reality with Smart Mobile Device. Proceedings of 2013 IEEE International Symposium on Mobile Cloud, Computing, and Service Engineering (IEEE Mobile Cloud 2013), Redwood City, 25-28 March 2013, 561-566. http://dx.doi.org/10.1109/sose.2013.57

[9] Lin, B.-S.P., Tung, L-P., Hsieh, I-C., Liu, T-H. and Chou, S-Y. (2015) The Design of Big Data Analytics for Testing \& Measurement and Traffic Flow on an Experimental 4G/LTE Network. Proceedings of IEEE WOCC 2015, Taipei, 2324 October 2015, 40-44. http://dx.doi.org/10.1109/wocc.2015.7346173

[10] Lin, B-S.P., Tung, L-P., Hsieh, I-C. and Chou, S-Y. (2015) Performance Estimation of MAR for Outdoor Navigation Applications on an 5G Mobile Broadband by Mobile Smart Devices. Proceedings of IEEE APWCS 2015, Singapore, 19-20 August 2015, 1-6.

[11] Lin, F.J., Ren, Y. and Cerritos, E. (2013) A Feasibility Study on Developing IoT/M2M Applications over ETSI M2M Architecture. Proceedings of the 1st International Workshop on Internet of Things Technologies (IoTT), Seoul, 15-18 December 2013, 558-563. http://dx.doi.org/10.1109/icpads.2013.100

[12] Chen, H.W. and Lin, F.J. (2014) Converging MQTT Resources in ETSI Standards Based M2M Platform. Proceedings of IEEE iThings, Taipei, 1-3 September 2014, 292-295. http://dx.doi.org/10.1109/ithings.2014.52

[13] Cerritos, E. and Lin, F.J. (2014) M2M-Enabled Real-Time Trip Planner. Proceedings of the 2nd International Workshop on Internet of Things Technologies (IoTT), Hsinchu, 16-19 December 2014, 886-891. http://dx.doi.org/10.1109/padsw.2014.7097902

[14] Lusiarta Putera, C.A. and Lin, F.J. (2015) Incorporating OMA Lightweight M2M Protocol in IoT/M2M Standard Architecture. Proceedings of IEEE World Forum on IoT, Milan, 14-16 December 2015, 559-564. 
http://dx.doi.org/10.1109/WF-IoT.2015.7389115

[15] Adrianto, D. and Lin, F.J. (2015) Analysis of Security Protocols and Corresponding Cipher Suites in ETSI M2M Standards. Proceedings of IEEE World Forum on IoT, Milan, 14-16 December 2015, 777-782. http://dx.doi.org/10.1109/wf-iot.2015.7389152

[16] Lin, F.J. and Chen, H. (2015) Improving Utilization and Customer Satisfaction of Parking Space with M2M Communications. Proceedings of IEEE World Forum on IoT, Milan, 14-16 December 2015, 465-470.

[17] Lin, F.J., Tsai, W., Cerritos, E., Lin, B.-T. and Hu, W.-H. (2015) Identification and Analysis of Charging Factors in M2M Communications. Proceedings of IEEE World Forum on IoT, Milan, 14-16 December 2015, 160-165. http://dx.doi.org/10.1109/wf-iot.2015.7389045 